\title{
The Diagnostic Accuracy and Postoperative Outcomes of Cervical Cancer Patients for MR-invisible or MR-visible Diagnosis of Combined T2- and Diffusion-weighted 3T MRI Using the External Phased-array Receiver
}

\author{
HYUN JIN ROH ${ }^{1}$, EUN BYEOL GO ${ }^{1}$, KYUNG BIN KIM ${ }^{2}$, JONG HWA LEE $^{3}$ and SANG HUN LEE ${ }^{1}$ \\ ${ }^{1}$ Department of Obstetrics and Gynecology, University of Ulsan College of Medicine, \\ Ulsan University Hospital, Ulsan, Republic of Korea; \\ ${ }^{2}$ Department of Pathology, University of Ulsan College of Medicine, \\ Ulsan University Hospital, Ulsan, Republic of Korea; \\ ${ }^{3}$ Department of Radiology, University of Ulsan College of Medicine, \\ Ulsan University Hospital, Ulsan, Republic of Korea
}

\begin{abstract}
Background/Aim: This study aimed to determine the diagnostic accuracy and postoperative outcomes of early-stage cervical cancer patients [2009 FIGO stages IA2IBI $(<2 \mathrm{~cm})]$ diagnosed with magnetic resonance $(M R)-$ invisible disease or MR-visible disease using the external phased-array receiver. Patients and Methods: Between 2007 and 2014, 110 patients with a FIGO clinical stage IA2-IBI $(<2 \mathrm{~cm})$ cervical cancer underwent primary surgical treatment after external array coil $T 2 W$ and $D W M R$ imaging following the diagnostic biopsy procedure. Results: The median histological size of MR-invisible vs. MR-visible diagnosis was $3 \pm 6.4 \mathrm{~mm}$ and $16 \pm 5.2 \mathrm{~mm}$. Eighty-five of the 110 patients had histologically residual tumor. The sensitivity, specificity, PPV, and NPV of tumor diagnosis were $63.5 \%, 92.0 \%, 96.4 \%$, and $42.6 \%$, respectively. Histological estimates of 54 (49.1\%) MR-invisible vs. 56 (50.9\%) MR-visible diagnoses were identified as 23 truenegative (TN) and 31 false-negative $(F N)$ vs. 54 true-positive (TP) and 2 false-positive (FP). The recurrence-free rate was $98.1 \%$ in the MR-invisible group and $91.1 \%$ in the MRvisible group. The overall survival rates were $100 \%$ and $92.9 \%$, respectively. Conclusion: A preoperative MRinvisible diagnosis in early-stage cervical cancer patients led
\end{abstract}

Correspondence to: Sang Hun Lee, Department of Obstetrics and Gynecology, Ulsan University Hospital, University of Ulsan College of Medicine, 877, Bangeojinsunhwando-ro, Dong-gu, Ulsan 44033, Republic of Korea. Tel: +82 0522508083, Fax: +82 0522507163, e-mail: med7311@ulsan.ac.kr

Key Words: Cervical cancer, false-negative diagnosis, magnetic resonance imaging, MR-invisible stage, MR-visible stage. to a high probability of FN and was associated with underdiagnosis.

In several developed countries, as a result of regular screening programs for pre-cancerous lesions, the incidence rate of cervical cancer has declined by as much as $65 \%$ over the past 40 years, and early-stage cervical cancer diagnosis with a small-volume disease is gradually increasing $(1,2)$.

Based on the current guidelines, clinicians recommend colposcopy through the "see and treat" approach for patients who have any abnormal cytology or a positive human papillomavirus (HPV) test in the screening process. If the colposcopic examination reveals an abnormal finding, the patients undergo conization or loop electrosurgical excisional procedure (LEEP).

Recent studies have reported that there is no residual disease in the surgical specimens of approximately $62-67 \%$ of patients undergoing radical trachelectomy (3). Meanwhile, magnetic resonance (MR)-invisible disease accounts for $25-39 \%$ of early stage cervical cancer patients, and 58-86\% of patients with MR invisible diagnosis had no residual tumor $(4,5)$.

Either diagnostic or therapeutic LEEP/conization can lead to incomplete excision and residual tumor extending to margins of the specimen (6). Accurate diagnosis of the size of the remaining tumor following LEEP/conization plays a significant role in the pre-operative management of stage IA2-Ib1 $(<2 \mathrm{~cm})$ cervical cancer patients (2009 FIGO staging) who should be considered for fertility-sparing surgery and less radical hysterectomy $(7,8)$. Therefore, the discriminating ability of residual disease has become a key prequisite for magnetic resonance imaging (MRI).

In theory, the SNR (signal-to-noise ratio) value would be double at 3T MRI compared with that at 1.5T MRI $(9,10)$. 
The improved SNR achieved with a high-field strength can be used to enhance the spatial resolution or reduce the imaging time. The relationship between the MR signal and image noise is represented as SNR. Therefore, a high SNR value is advisable on MRI. To improve the SNR value of the uterine cervix, the spatial resolution of the MR images may be enhanced by the use of endovaginal receiver coils adjacent to the region of interest $(6,11,12)$.

Despite these advantages, the use of endovaginal coils may be limited in practice because of the discomfort induced by inserting the coil into the vagina and the difficulty in correctly positioning the coil in the vagina for precise cervical MR scanning. As an alternative measure, a pelvic phased-array coil that can be quickly and universally used is needed.

The goal of this study was to determine the diagnostic accuracy and postoperative outcomes of preoperative MRI using external phased-array receiver coils for early-stage cervical cancer patients [2009 FIGO stages IA2-IB1 $(<2$ $\mathrm{cm})$ ] diagnosed with MR-invisible disease or MR-visible disease.

\section{Patients and Methods}

Study population. After obtaining approval (UUH 2019-05-027) from the institutional review board, the clinical and MRI data and pathological reports from January 2007 to December 2014 at the Ulsan University Hospital were retrospectively reviewed.

Inclusion criteria were as follows: 1) patients with biopsyconfirmed stage IA2-IB $1(<2 \mathrm{~cm})$ cervical cancer, based on the 2009 FIGO system, detected by punch biopsy and/or LEEP/conization procedure; 2) patients who underwent preoperative MRI at least 7 days after a biopsy; 3) patients with cancer classified as squamous, adenocarcinoma or adenosquamous carcinoma; and 4) patients who underwent either fertility-sparing surgery (radical trachelectomy pelvic \pm para-aortic lymphadenectomy) or nonfertilitysparing surgery (type $\mathrm{C} 1$ or $\mathrm{C} 2$ hysterectomy and pelvic \pm paraaortic lymphadenectomy). Patients were excluded if they had received previous treatment with radiotherapy and/or chemotherapy for cervical cancer. Consequently, 110 patients were enrolled in this study (Figure 1).

Imaging procedure. All patients were scanned by MRI using phased-array techniques involving pelvic or torso phased-array coils at 3T (Intera Achieva 3T, Philips Medical Systems, Best, the Netherlands). The SENSE XL Torso coil 16-element phased array was generally applicable (Philips Medical Systems). A flexible volume coil was designed to combine anterior and posterior sections. Each section consisted of 4 upper and four lower elements. The coil dimensions were $74 \mathrm{~mm} \times 558 \mathrm{~mm} \times 600 \mathrm{~mm}$ per coil half. Each element used a Z coverage of $22.5 \mathrm{~cm}$. Images were obtained using the following parameters: for the pelvic region, transverse T1weighted, fast spin-echo (FSE) sequence: time of repetition (TR)/ time to echo (TE), 600/10 ms; slice thickness (ST), $5 \mathrm{~mm}$; interslice gap, $0.5 \mathrm{~mm}$; field of view (FOV), $20 \mathrm{~cm} \times 20 \mathrm{~cm}$; matrix, 400 $\times 284$; echo train length (ETL), 5; number of excitations (NEX), 2; no fat saturation; bandwidth, $21.87 \mathrm{kHz}$; for the transverse T2- weighted FSE sequence: TR/TE, 5000/90 ms; ST, $5 \mathrm{~mm}$ with no interslice gap, $0.5 \mathrm{~mm}$; FOV, $20 \mathrm{~cm} \times 20 \mathrm{~cm}$, matrix, $286 \times 286$; ETL, 20; NEX, 2; no fat saturation; bandwidth, $22.99 \mathrm{kHz}$; for the sagittal T2-weighted FSE sequence: TR/TE, 4000/90 ms; ST, 6 mm; interslice gap, $1 \mathrm{~mm}$; FOV, $19 \mathrm{~cm} \times 19 \mathrm{~cm}$; matrix, $316 \times 310$; ETL, 20; NEX, 3; no fat saturation; bandwidth, $23.67 \mathrm{kHz}$; and for the coronal T2-weighted, FSE sequence: TR/TE, 5000/90 ms; ST, 4 $\mathrm{mm}$; no interslice gap; FOV, $20 \mathrm{~cm} \times 20 \mathrm{~cm}$; matrix, 332×316; ETL, 20; NEX, 2; no fat saturation; bandwidth, $25.34 \mathrm{kHz}$. Single-shot diffusion-weighted (DW) echo-planar images (TR/TE 5650/64, acquisition matrix $=124 \times 124 \mathrm{FOV}, 25 \mathrm{~cm} \times 25 \mathrm{~cm}$ ) employing b values of 0 and $800 \mathrm{~s} / \mathrm{mm}^{2}$ were obtained in three planes to match the $\mathrm{T} 2-\mathrm{W}$ images, with in-plane resolution $=1.25 \mathrm{~mm}$ and voxel size $=3.15 \mathrm{~mm}^{3}\left(0.41 \mathrm{~mm}^{3}\right.$ reconstructed $)$. Thirty $4 \mathrm{~mm}$ thick sections provided coverage of the cervix (acquisition time 4 min 33 s). Isotropic apparent diffusion coefficient (ADC) maps were generated with a Phillips system software using all $b$ values.

Image analysis. All MR images stored in the picture archiving and communication system (PACS) were reviewed by a radiologist (J.W.L.) with more than 10 years of experience in the field of gynecological cancer imaging, who interpreted the images as positive or negative for the presence of a tumor based on the integrated information of T2-W and DW images in combination with ADC measurement.

On the integrated sequences, if there was no abnormally located signal intensity, the images were defined as MR-invisible diagnosis. In contrast, the images were assessed as MR-visible diagnosis if the signal intensity was higher than the normal low signal intensity of the cervical stroma.

The largest tumor diameter visualized in the anterior-posterior, and transverse and craniocaudal directions were also determined by axial, sagittal and coronal $\mathrm{T} 2-\mathrm{W}$ images. The diameter-based tumor volume was calculated using a formula that assumes an ellipsoid configuration, $V=4 / 3 \pi \times a / 2 \times b / 2 \times c / 2=052 \times a b c$. The anteroposterior, transverse, and craniocaudal tumor dimensions are listed in a, b, and c, and they are the orthogonal measurements. The distance from the tumor to the internal os was recorded on the sagittal T2-weighted images.

Histopathological analysis. Surgical specimens for histological diagnosis were collected according to a standard protocol in our institute. An independent pathologist (K.B.K.) reviewed the wholemount $H \& E$ stained slides following formalin fixation and paraffin embedding of surgical specimens. The cutting of specimens was performed by conventional longitudinal sectioning along the vertical axis connecting the internal os of the uterine cervix and the vaginal resection margin after amputation at or near the internal os of the uterus (13).

Tumor size refers to the maximum tumor diameter (in $\mathrm{mm}$ ) measured along the surface epithelium perpendicular to stromal infiltration. Tumor depth of invasion (in $\mathrm{mm}$ ) was measured perpendicularly from the basement membrane of the surface epithelium. In the case of multifocal disease, the dimension of the largest focus was used for analysis. If the residual tumor was observed in the hysterectomy specimen, the summation of the depth of invasion from the corresponding site in the conization specimen was performed to give the best estimation.

The overall stage was determined according to FIGO 2009 and the recently updated 8th edition AJCC staging system (Table I). 


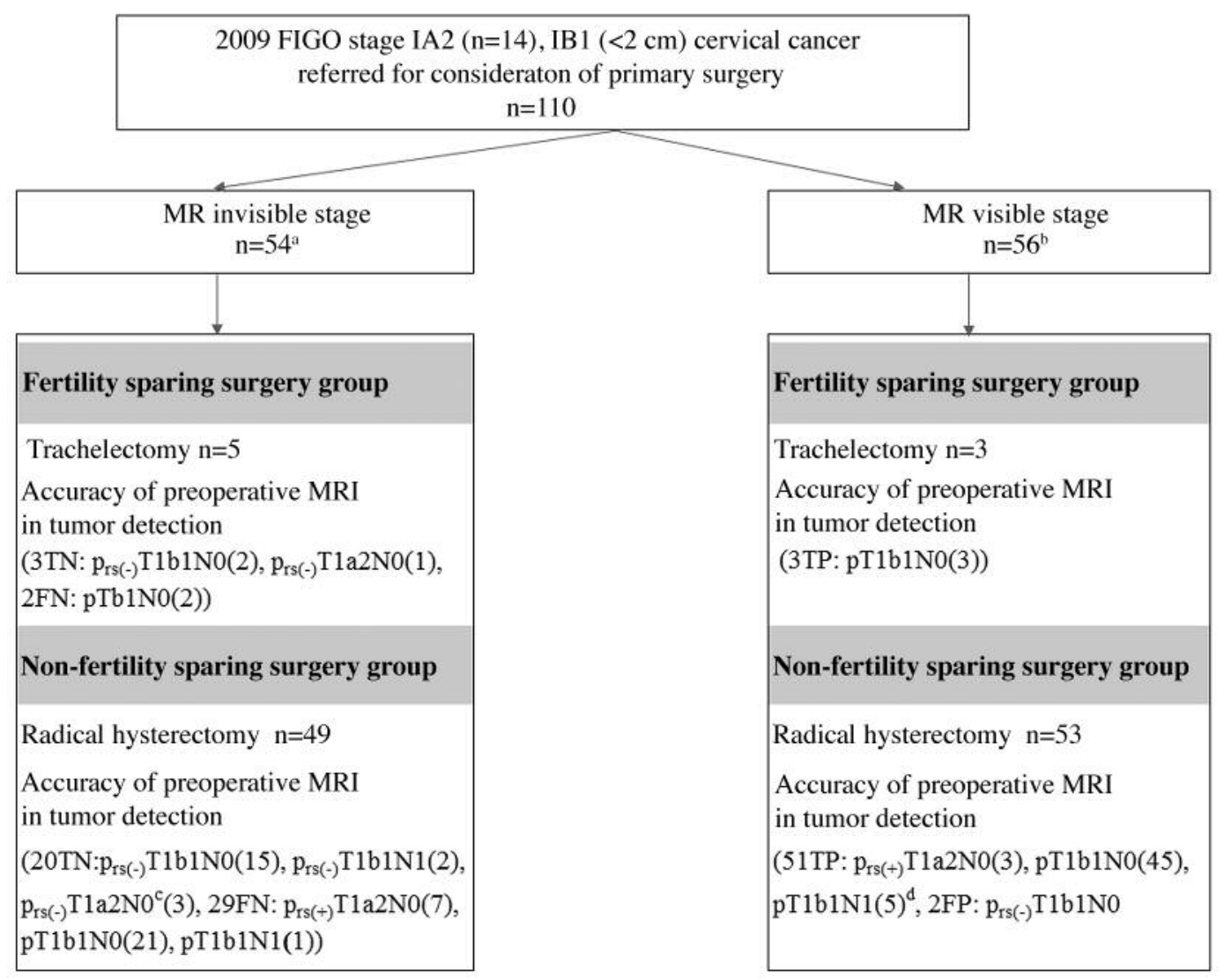

Figure 1. Study population. ${ }^{a} 1$ case recurrence; ${ }^{b} 5$ cases recurrence; ${ }^{c} 1$ case recurrence only at lung; ${ }^{d} 5$ of 5 cases recurrence (1 of 5 cases: pelvic outside recurrence; 4 of 5 cases: pelvic inside recurrence; 1 of 5 cases: conversion to non-fertility sparing surgery due to lymph node metastasis during fertility-sparing surgery). pT: Primary tumor; $p$ T0: no evidence of primary tumor; $p T 1$ : cervical carcinoma confined to uterus (extension to corpus should be disregarded); pT1a2: measured stromal invasion of more than $3.0 \mathrm{~mm}$ and not more than $5.0 \mathrm{~mm}$; pT1b1: clinically visible lesion $2.0 \mathrm{~cm}$ or less in greatest dimension. $p N$ : regional lymph nodes; $r s$ : presence or absence of residual specimen after surgery including hysterectomy, trachelectomy among biopsy-proven (punch biopsy or a conization or LEEP procedure) cervical cancer IA2-IB1; TN: true negative; FN: false negative; TP: true positive; FP: false positive.

This study documented the absence or presence of residual masses in surgical specimens after punch biopsy and/or LEEP/conization procedure. Depending on the existence of a postoperative residual tumor, 2009 FIGO IA2, and IB1 were analyzed and classified as $\mathrm{p}_{\mathrm{rs}(-)} \mathrm{T} 1 \mathrm{~A} 2$ stage (rs: residual specimen), $\mathrm{p}_{\mathrm{rs}(-)} \mathrm{T} 1 \mathrm{~B} 1$ stage in postoperative negative residual tumor and as $\mathrm{p}_{\mathrm{rs}(+)} \mathrm{T} 1 \mathrm{~A} 2$ stage in the postoperative positive residual tumor (Figure 2$)(14,15)$.

Statistical analyses. The patients were allocated into two groups (the MR-invisible group and the MR-visible group). Continuous variables were analyzed using Student's $t$-tests and the MannWhitney U-statistic, whereas the $\chi^{2}$ test and Fisher's exact test were used for categorical variables.

The progression-free survival (PFS) and overall survival (OS) rates between the groups were estimated using Kaplan-Meier survival curves. Values of $p<0.05$ were considered statistically significant. SPSS software (version 21.0; SPSS Inc., Chicago, IL, USA) was used for statistical analyses.

\section{Results}

The characteristics of the patients included in this study are shown in Table II. A total of 110 patients satisfied the inclusion criteria and were examined in this study (Figure 1).

There was a significant difference in age among the 54 patients with MR-invisible diagnosis [median: 45.5 \pm 17.0 (range=22-77) years] and the 56 patients with MR-visible diagnosis [median: 49.5 \pm 14.0 (range $=24-85$ ) years, $p=0.046$ ].

Of the 110 patients referred for primary surgery, 54 (49.1\%) patients had no visible tumor diagnosis on MRI [median histological size: $3 \pm 5.4(0-19) \mathrm{mm}$ ]. Fifty-six (50.9\%) patients had an MR-visible diagnosis [median histological size: $14 \pm 5.1(0-19) \mathrm{mm}]$. A total of $25(22.7 \%)$ patients had histologically no residual tumor. Overall, $42.6 \%$ in the MR-invisible diagnosis had no residual tumor, and 
Table I. Comparison of MRI staging to surgical-pathologic stage 2009 FIGO surgical-pathological stage of cancer of the cervix.

\begin{tabular}{|c|c|c|c|c|c|c|c|}
\hline & \multicolumn{2}{|c|}{ Stage IA2 } & \multirow{2}{*}{$\begin{array}{c}\text { Stage IB1 } \\
\mathrm{B}=\mathrm{p}_{\mathrm{rs}(-)} \mathrm{T} 1 \mathrm{~B} 1\end{array}$} & \multirow{2}{*}{$\begin{array}{l}\text { Stage IB1 } \\
\mathrm{C}=\mathrm{pT} 1 \mathrm{~B} 1\end{array}$} & \multirow{2}{*}{$\begin{array}{l}\text { Stage IIA1 } \\
\mathrm{D}=\mathrm{pT} 2 \mathrm{~A} 1\end{array}$} & \multirow{2}{*}{$\begin{array}{l}\text { Stage IIB2 } \\
E=p T 2 B 1\end{array}$} & \multirow{2}{*}{$\begin{array}{l}\text { Positive nodes } \\
\text { outside true pelvis }\end{array}$} \\
\hline & $\mathrm{O}=\mathrm{p}_{\mathrm{rs}(-)} \mathrm{T} 1 \mathrm{~A} 2$ & $\mathrm{~A}=\mathrm{p}_{\mathrm{rs}(+)} \mathrm{T} 1 \mathrm{~A} 2$ & & & & & \\
\hline MRI invisible diagnosis & 4 & 7 & $19^{\mathrm{a}}$ & $24^{\mathrm{b}}$ & 0 & 0 & 0 \\
\hline MRI visible diagnosis & 0 & 3 & 2 & $48^{\mathrm{c}}$ & 1 & 2 & 1 \\
\hline
\end{tabular}

a2 cases lymph node metastasis; ${ }^{b} 1$ case lymph node metastasis; ${ }^{\mathrm{c}} 6$ cases lymph node metastasis. rs: Presence or absence of residual surgical specimen after surgery including radical hysterectomy, trachelectomy; FIGO: International Federation of Gynecology and Obstetrics; O: This study suggested that 2009 FIGO stage IA2 diagnosed by LEEP/conization being classified as $\mathrm{p}_{\mathrm{rs}(+)} \mathrm{T} 1 \mathrm{~A} 2$ in postoperative negative residual tumor cases; A: This study suggested that the 2009 FIGO stage IA2 diagnosed by LEEP/conization classified as $\mathrm{p}_{\mathrm{rs}(+)} \mathrm{TA} 2$ if there is no residual tumor at the final surgical specimen; B: 2009 FIGO stage IB1 also was stratified as a $\mathrm{p}_{\mathrm{rs}(-)} \mathrm{T} 1 \mathrm{~B} 1$ stage if there is no residual tumor at the final surgical specimen; C: 2009 FIGO stage IB1 also was stratified as a $\mathrm{p}_{\mathrm{rs}(+)} \mathrm{T} 1 \mathrm{~B} 1$ stage if there is residual tumor at the final surgical specimen; D: Carcinoma extends from the cervix to involve vagina with $<4 \mathrm{~cm}$ in a great dimension; E: Carcinoma extends from the cervix and involve parametrial tissue but not up to the pelvic wall.

$3.6 \%$ in the MR-visible diagnosis had no residual tumor. A total of $85(77.3 \%)$ patients had a residual tumor (median histological volume, size: $0.3 \pm 0.64 \mathrm{~cm}^{3}$ ).

The sensitivity, specificity, positive predictive value (PPV) and negative predictive value (NPV) for detecting a tumor in 110 patients treated for 2009 FIGO stage IA2-IBI $(<2 \mathrm{~cm})$ cancer were $63.5 \%, 92.0 \%, 96.4 \%$, and $42.6 \%$, respectively (Table III).

An analysis was performed between the patients in the MR-visible group with positive results and those in the MRinvisible group with negative results from the diagnostic test. Of the 54 patients with MR-invisible diagnosis, 15 did not undergo, and 39 did undergo LEEP/conization prior to imaging, which demonstrated 23 true-negative diagnoses (TNs) and 31 false-negative diagnoses (FNs). A total of 56 patients with MR-visible diagnosis comprised 31 patients without and 25 patients with LEEP/conization prior to imaging and demonstrated 54 true-positive diagnoses (TPs) and 2 false-positive diagnoses (FPs).

In the MR-invisible group, the difference in the number of FNs between patients who did and did not undergo LEEP/conization [17 FNs (43.6\%) with LEEP/conization and 14 FNs $(93.3 \%)$ without LEEP/conization] was significant $(p=0.0015$, Table III). However, in the MR-invisible subgroup with residual tumor, the difference in the number of FNs between patients who did and did not undergo LEEP/conization [17 FNs $(100 \%)$ with LEEP/conization and 14 FNs $(100 \%)$ without LEEP/conization] was not significant $(p=1.000)$.

The maximum histological dimension of the tumors among those with FNs was $13 \mathrm{~mm}$ in the no LEEP/conization group and $19 \mathrm{~mm}$ in the LEEP/conization group.

In the MR-visible group, the difference in the number of FPs between patients who did and did not undergo the procedure $[2$ FPs $(8 \%)$ with and 0 FPs $(0 \%)$ without the procedure] was also not significant ( $p=0.195$, Table III).
Table II. Characteristics of patients with 2009 FIGO stage IA2-IB1 $(<2$ cm) cervical cancer.

\begin{tabular}{|c|c|c|c|}
\hline \multirow[t]{2}{*}{ Variable } & \multicolumn{2}{|c|}{$\begin{array}{l}2009 \text { FIGO stage } \\
\text { IA2-IB1 cervical cancer }\end{array}$} & \multirow[t]{2}{*}{$p$-Value } \\
\hline & $\begin{array}{l}\text { MR invisible } \\
(\mathrm{n}=54)\end{array}$ & $\begin{array}{l}\text { MR-visible } \\
\quad(\mathrm{n}=56)\end{array}$ & \\
\hline \multicolumn{4}{|l|}{ Age, years $\mathrm{a}$} \\
\hline Median, range & $\begin{array}{l}45.5 \pm 17.0 \\
(22-77)\end{array}$ & $\begin{array}{l}49.5 \pm 14.0 \\
(24-85)\end{array}$ & 0.046 \\
\hline Procedure & & & 0 \\
\hline Colposcopic biopsy, n (\%) & $15(27.8 \%)$ & $31(55.4 \%)$ & \\
\hline LEEP/conization, n (\%) & $39(72.2 \%)$ & $25(44.6 \%)$ & \\
\hline 2009 FIGO staging & & & 0.023 \\
\hline IA2 & $11(20.3 \%)$ & $3(5.4 \%)$ & \\
\hline IB1 & $43(79.7 \%)$ & $53(94.6 \%)$ & \\
\hline Histologic classification & & & 0.693 \\
\hline Squamous cell carcinoma, $\mathrm{n}(\%)$ & $33(61.1 \%)$ & $36(64.3 \%)$ & \\
\hline Adenocarcinoma, n (\%) & $19(35.2 \%)$ & $18(32.1 \%)$ & \\
\hline Adeno-squamous, n (\%) & $2(3.7 \%)$ & $2(3.6 \%)$ & \\
\hline \multicolumn{4}{|l|}{ Tumor marker } \\
\hline $\mathrm{SCC} \operatorname{Ag}^{\mathrm{a}}(\mathrm{ng} / \mathrm{ml})$ & $\begin{array}{l}0.8 \pm 0.7 \\
(0.1-3.1)\end{array}$ & $\begin{array}{l}0.8 \pm 0.65 \\
(0.4-28.4)\end{array}$ & 0.598 \\
\hline
\end{tabular}

Mann-Whitney test was used to compare age and squamous cell carcinoma antigen. Fisher exact test was used to compare types of biopsy or histologic types of cervical cancer. ${ }^{\text {DData }}$ are given as median \pm Inter Quartile Range (IQR) (range).

Of the 14 patients with stage IA 2 disease confirmed by LEEP/conization, ten patients ( 7 in the MR-invisible group, 3 in the MR-visible group) had residual tumor on the histological specimens after surgery. However, all residual cases had superficial stromal invasion.

In the MR-invisible group, 5 patients underwent radical trachelectomy, and 49 patients underwent radical hysterectomy; among the 56 patients in the MR-visible group, 3 patients 

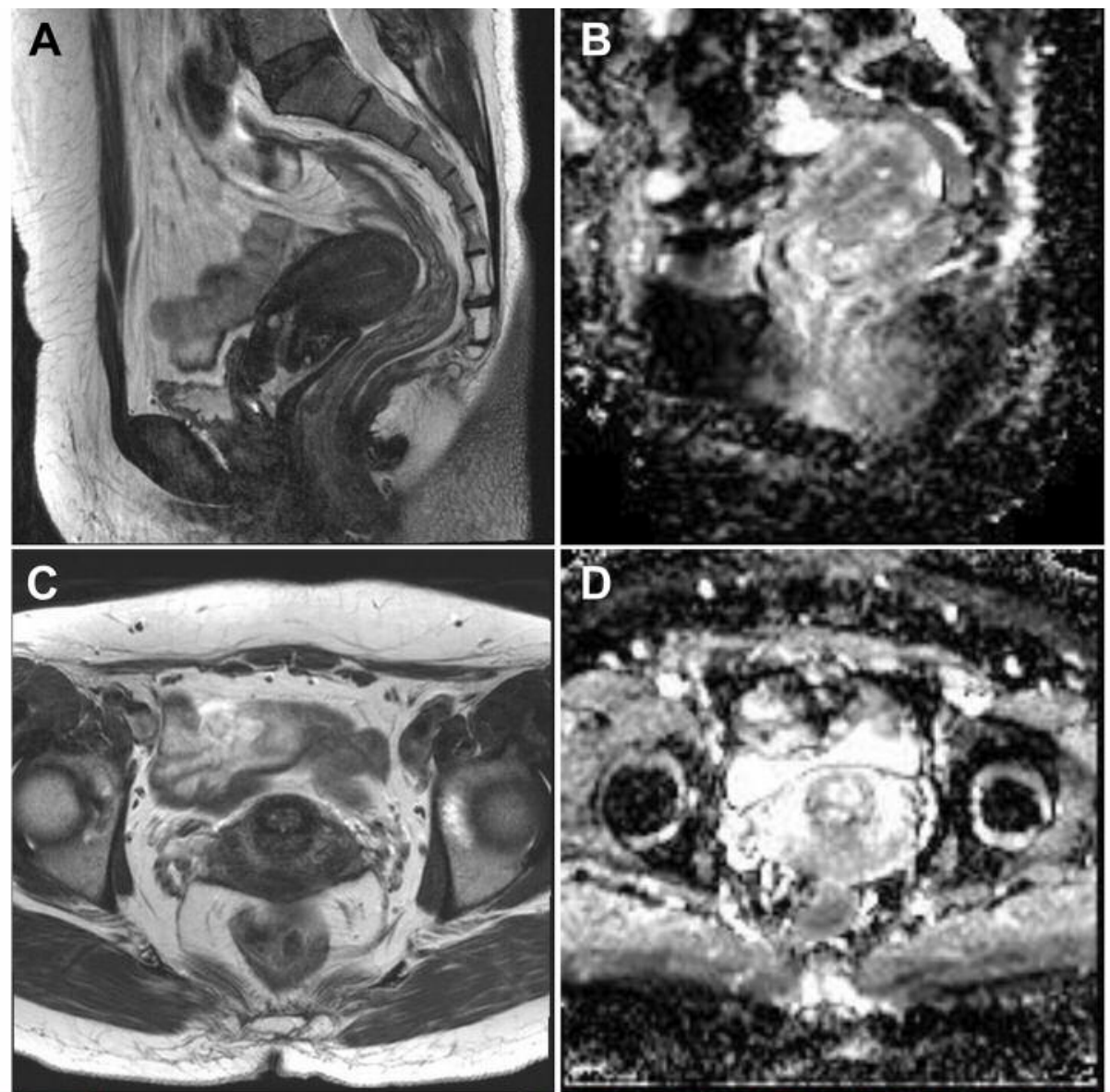

E
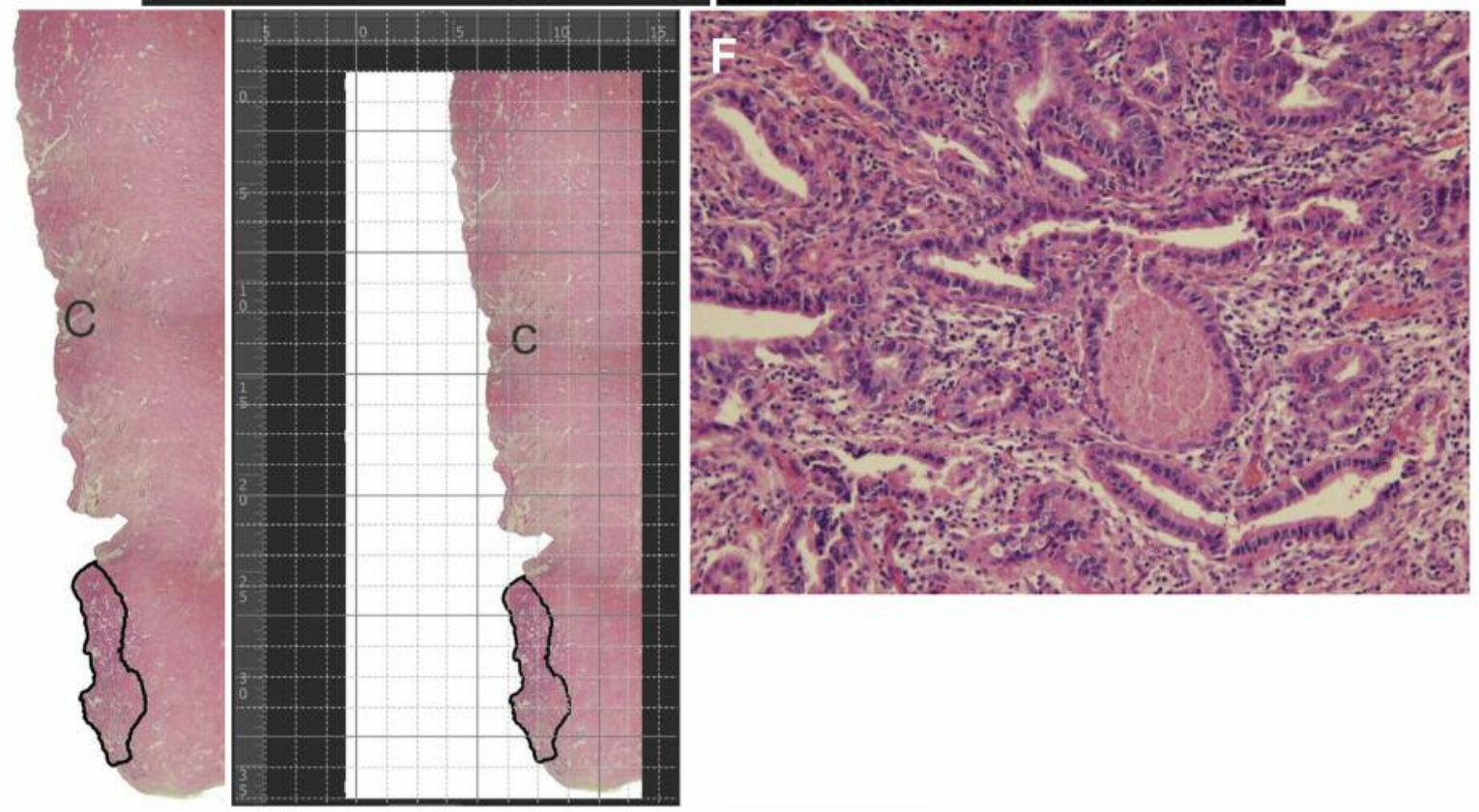

Figure 2. 2009 FIGO Stage IA2 cervical adenocarcinoma in a 46-year-old woman with a punch biopsy prior to MRI. (A) Sagittal T2-w image, (B) sagittal ADC map, (C) axial T2-w images, (D) axial ADC map, all images show no gross cancerous lesion of the uterine cervix. At histopathological findings for radical hysterectomy, $9 \times 9 \times 4 \mathrm{~mm}$ residual tumor in uterine cervix was found and had a superficial stromal invasion, and (E) longitudinal section showing a tumor-to-internal os distance was photographed, and $24 \mathrm{~mm}$ calculated. $(F)$ The tumor is composed of irregular mucin-poor glands that exhibit a relatively well-formed structure but an infiltrative pattern. Mitotic activity is low. C: Cervical wall. 
Table III. Sensitivity, specificity, positive and negative predictive values for identifying invasive cervical carcinoma using T2-W and ADC maps in patients without or with previous LEEP/conization biopsies.

\begin{tabular}{|c|c|c|c|c|c|c|c|c|c|c|c|c|}
\hline & $\mathrm{TP}$ & \multicolumn{2}{|c|}{$\mathrm{TN}$} & FP & FN & Sensitivity (\%) & \multicolumn{2}{|c|}{ Specificity (\%) } & \multicolumn{2}{|c|}{ PPV (\%) } & \multicolumn{2}{|r|}{ NPV $(\%)$} \\
\hline LEEP/Conization $(\mathrm{n}=64)$ & 23 & \multicolumn{2}{|c|}{22} & 2 & 17 & $57.5(42.2-72.9)$ & \multicolumn{2}{|c|}{$91.7(80.6-100.0)$} & \multicolumn{2}{|c|}{$92.0(81.4-100)$} & \multicolumn{2}{|c|}{$56.4(40.9-72.0)$} \\
\hline \multirow{3}{*}{ Non LEEP/Conization $(n=46)$} & 31 & \multicolumn{2}{|c|}{1} & 0 & 14 & $68.9(55.4-82.4)$ & \multicolumn{2}{|c|}{$100(100-100)$} & \multicolumn{2}{|c|}{$100(100-100)$} & \multicolumn{2}{|c|}{$6.7(0.1-19.3)$} \\
\hline & 54 & \multicolumn{2}{|c|}{23} & 2 & 31 & $63.5(53.3-73.8)$ & \multicolumn{2}{|c|}{$92.0(81.4-100.0)$} & $96.4(91.6$ & $6-100.0)$ & \multicolumn{2}{|c|}{$42.6(29.4-55.8)$} \\
\hline & $\mathrm{TP}$ & $\mathrm{TN}$ & FP & $\mathrm{FN}$ & Disease $(+)$ & Disease $(-)$ & Sensitivity $(\%)$ & Spec & cificity $(\%)$ & PPV & $(\%)$ & NPV (\%) \\
\hline \multicolumn{13}{|l|}{ Visible MR group } \\
\hline Non-LEEP/Conization $(\mathrm{n}=31)$ & 31 & & & & 54 & 2 & & & & & & \\
\hline LEEP/Conization $(\mathrm{n}=25)$ & 23 & & 2 & & & & 63.5 & & 92.0 & 96. & & 42.6 \\
\hline Invisible MR group & & & & & & & $(53.3-73.8)$ & & $.4-100.0)$ & $(91.6-1)$ & $00.0)$ & $(29.4-55.8)$ \\
\hline Non-LEEP/Conization( $\mathrm{n}=15)$ & & 1 & & 14 & 31 & 23 & & & & & & \\
\hline LEEP/Conization $(\mathrm{n}=39)$ & & 22 & & 17 & & & & & & & & \\
\hline
\end{tabular}

TP: True positive; TN: true negative; FP: false positive; FN: false negative; PPV: positive predictive value; NPV: negative predictive value.

underwent radical trachelectomy, and 53 patients underwent radical hysterectomy.

When comparing the MR-invisible and MR-visible groups in terms of surgical specimens, the median pathological tumor sizes were $3 \pm 5.4(0-19) \mathrm{mm}$ and $14 \pm 5.1(0-19) \mathrm{mm}$, respectively ( $p=0.000$; Table IV). The median depth of invasion was $16.7 \pm 36.8 \%$ and $50 \pm 41.0 \%$, respectively $(p<0.001)$. The superficial stromal invasion was $68.5 \%(37 / 54)$ and $26.8 \%(15 / 56)$, respectively. The middle stromal invasion was $27.8 \%(15 / 54)$ and $42.8 \%(24 / 56)$, respectively. The deep stromal invasion was $3.7 \%(2 / 54)$ and $30.4 \%(17 / 56)$, respectively. Lymphovascular invasion was shown in $5.6 \%$ (3/54) and 26.8\% (15/56) of the cases, respectively. Parametrial invasion and vaginal invasion were not demonstrated in any of the MR-invisible tumors, but parametrial invasion or vaginal involvement was indicated in $3.6 \%(2 / 56)$ of MR-visible tumors, respectively. Lymph node metastasis was detected in $5.6 \%(3 / 54)$ of the patients in the MR-invisible group and $10.7 \%(6 / 56)$ of the patients in the MR-visible group.

This study assessed the distance between the tumor and the internal os at the final histologic specimen as well as MR imaging. In the MR-invisible group, the distance between the tumor and the internal os ranged from 15.6 to $35.1 \mathrm{~mm}$ (median: $26.5 \pm 3.7 \mathrm{~mm}$ ) on MR imaging and from $4.2 \mathrm{~mm}$ to $26.0 \mathrm{~mm}$ (median: $11.9 \pm 6 \mathrm{~mm}$ and $9.7 \pm 4.3 \mathrm{~mm}$ ) on the histologic specimen.

At the final histological specimens of the MR-invisible group, tumor-to-internal os distance identified 2 patients (1 superficial, 1 middle stromal invasion) at $5 \mathrm{~mm}$ or less (Figure 2), 14 patients [8 superficial, 5 middle ( 2 of 5 cases: $>50 \%$ stromal invasion) and 1 deep stromal invasion] at 5.1$9.9 \mathrm{~mm}$ and 38 patients [29 superficial, 8 middle ( 1 of 8 case: $>50 \%$ stromal invasion) and 1 deep stromal invasion] at $10 \mathrm{~mm}$ or more (Figures 3 and 4 ).
Of 38 patients with a tumor-to-internal os distance of 10 $\mathrm{mm}$ or more in invisible MR diagnosis, 3 patients had lymph node metastasis (2 cases with no tumor at surgical specimens, and 1 case of middle stromal invasion with $<50 \%$ ), and no parametrial involvement and vagina invasion.

Recurrent tumors were observed in $1.9 \%$ (1/54) of the MR-invisible patients and $8.9 \%(5 / 56)$ of the MR-visible patients on follow-up CT. The recurrence-free survival rate was $98.1 \%(53 / 54)$ in the MR-invisible group and $91.1 \%$ $(51 / 56)$ in the MR-visible group ( $p=0.058$; Figure $5 \mathrm{~A})$. The OS rate was $100 \%(54 / 54)$ in the MR-invisible group and $92.9 \%(52 / 56$ cases $)$ in the MR-visible group ( $p=0.048$; Figure 5B).

\section{Discussion}

A high level of inaccuracy was identified upon visualizing the true characteristics of stage IA2-IB1 $(<2 \mathrm{~cm})$ disease that appeared to be invisible on MR images due to its spatial resolution. When we considered the inclusion criteria mentioned above regarding radical trachelectomy, up to $18.5 \%$ of patients with an invisible diagnosis on MRI could have been converted to a more radical treatment than to radical trachelectomy. Lastly, the recurrence-free and overall longterm survival rates of patients with a MR-invisible diagnosis were better than in those with a MR-visible diagnosis.

In theory, the SNR value would be double at 3T MRI compared with that at $1.5 \mathrm{~T}$ MRI $(9,10)$. The improved SNR achieved with high-field strength can be used to enhance the spatial resolution or reduce the imaging time.

The relationship between the MR signal and image noise is represented as SNR. Therefore, a high SNR value is advisable on MRI. Improvement in the SNR value can be influenced by an increase in the value of the following 
Table IV. Pathologic comparison of MR invisible and visible cancers.

\begin{tabular}{|c|c|c|c|}
\hline & \multicolumn{2}{|c|}{2009 FIGO stage IA2-IB1 $(<2 \mathrm{~cm})$ cervical cancer } & \multirow[t]{2}{*}{$p$-Value } \\
\hline & MR invisible $(\mathrm{n}=54)$ & MR-visible $(n=56)$ & \\
\hline Tumor size, mm & & & 0.0000 \\
\hline Median(range) $)^{\mathrm{a}}$ & $3.0 \pm 5.4(0-19)$ & $14.0 \pm 5.1(0-19)$ & \\
\hline Histologic grade & & & 0.6060 \\
\hline Grade 1 & $20(37 \%)$ & $14(25 \%)$ & \\
\hline Grade 2 & $22(40.7 \%)$ & $27(48.2 \%)$ & \\
\hline Grade 3 & $5(9.3 \%)$ & $12(21.4 \%)$ & \\
\hline Undetermined & $7(13 \%)$ & $3(5.4 \%)$ & \\
\hline Depths of stromal invasion, $\%$ a & $16.7 \pm 36.8(0-75)$ & $50.0 \pm 41.0(0-100)$ & $<0.001$ \\
\hline Superficial stromal invasion & $37(68.5 \%)$ & $15(26.8 \%)$ & \\
\hline Middle stromal invasion & $15(27.8 \%)$ & $24(42.8 \%)$ & \\
\hline Deep stromal invasion & $2(3.7 \%)$ & $17(30.4 \%)$ & \\
\hline \multicolumn{4}{|c|}{ Distance between the tumor and internal os at MR imaging (mm) } \\
\hline $0-5$ & 0 & 5 & \\
\hline $5.1-9.9$ & 0 & 26 & \\
\hline$\geq 10$ & 54 & 25 & \\
\hline \multicolumn{4}{|c|}{ Distance between the tumor and internal os at final histology $(\mathrm{mm})$} \\
\hline $0-5$ & $2(3.7 \%)$ & $12(21.4 \%)$ & \\
\hline $5.1-9.9$ & $14(25.9 \%)$ & $32(57.1 \%)$ & \\
\hline$\geq 10$ & $38(70.4 \%)$ & $12(21.5 \%)$ & \\
\hline No residual tumor & $23(42.6 \%)$ & $2(3.6 \%)$ & \\
\hline Parametrial involvement & $0(0 \%)$ & $2(3.6 \%)$ & 0.1610 \\
\hline Lymphovascular space invasion & $3(5.6 \%)$ & $15(26.8 \%)$ & 0.0030 \\
\hline Vaginal invasion & $0(0 \%)$ & $2(3.6 \%)$ & 0.1610 \\
\hline Lymph node metastasis & $3(5.6 \%)$ & $6(10.7 \%)$ & 0.3240 \\
\hline
\end{tabular}

Mann-Whitney test was used to compare age and squamous cell carcinoma antigen. Fisher exact test was used to compare types of biopsy or

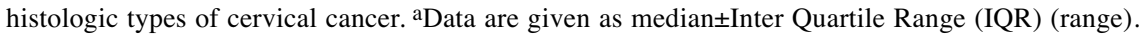

factors: ST, FOV, magnetic field strength, the strength of the transmission and receiver coil radiofrequency (RF), and TR, which can be influenced by a decrease in the value of the following factors: receiver bandwidth, size of the image matrix, and TE. To improve the SNR value of the uterine cervix, the spatial resolution of the MR images may be enhanced by the use of endovaginal receiver coils adjacent to the region of interest $(6,11,12)$.

A handful of studies have shown that endovaginal T2-W MRI is superior to pelvic phased-array coil MRI in detecting small lesions in patients who have undergone conization or LEEP. Charles-Edwards et al. have reported on the use of these imaging methods and showed a $100 \%$ sensitivity and specificity for detecting $5.3 \mathrm{~mm}$ tumors, regardless of LEEP/conization procedures (8).

Two recent studies on the use of endovaginal coils have demonstrated the efficacy of preoperative MRI using external coils. Lakhman Y et al. have identified the values of high sensitivity and specificity for patients undergoing radical trachelectomy (16). Downey K et al. have identified the efficacy of endovaginal versus external-array coil T2-W plus DWI for detecting small cervical tumors and reported that sensitivity, specificity, PPV, NPV were $78.2 \%, 89.5 \%$, $89.5 \%, 73.9 \%$, respectively, for T2-W plus DW endovaginal MRI, and 66.7\%, 94.7\%, 94.1\%, 69.2\%, respectively, for T2W plus DW external-array coil MRI (17).

In this study, the sensitivity, specificity, PPV, and NPV for detecting tumors were $63.5 \%, 92.0 \%, 96.4 \%$, and $42.6 \%$, respectively.

Several studies have reported that MRI, regardless of imaging techniques, was useful in detecting the presence of tumors in the endocervix and to guide decisions regarding fertility-sparing surgery $(16,18)$.

Particularly for deciding on the surgical radicality for patients who wish to preserve fertility, weighing the reliability of an MR-invisible tumor stage before undergoing radical trachelectomy is essential.

Previous investigators and NCCN (National Comprehensive Cancer Network) guidelines have suggested that candidates for fertility-preserving radical trachelectomy must be carefully selected and considered using the following criteria: 1) tumor $<2 \mathrm{~cm}, 2$ ) a clearance of $5 \mathrm{~mm}$ or greater at distance between the tumor and the internal os, and 3) cervical stromal invasion less than $50 \%(19-21)$. 

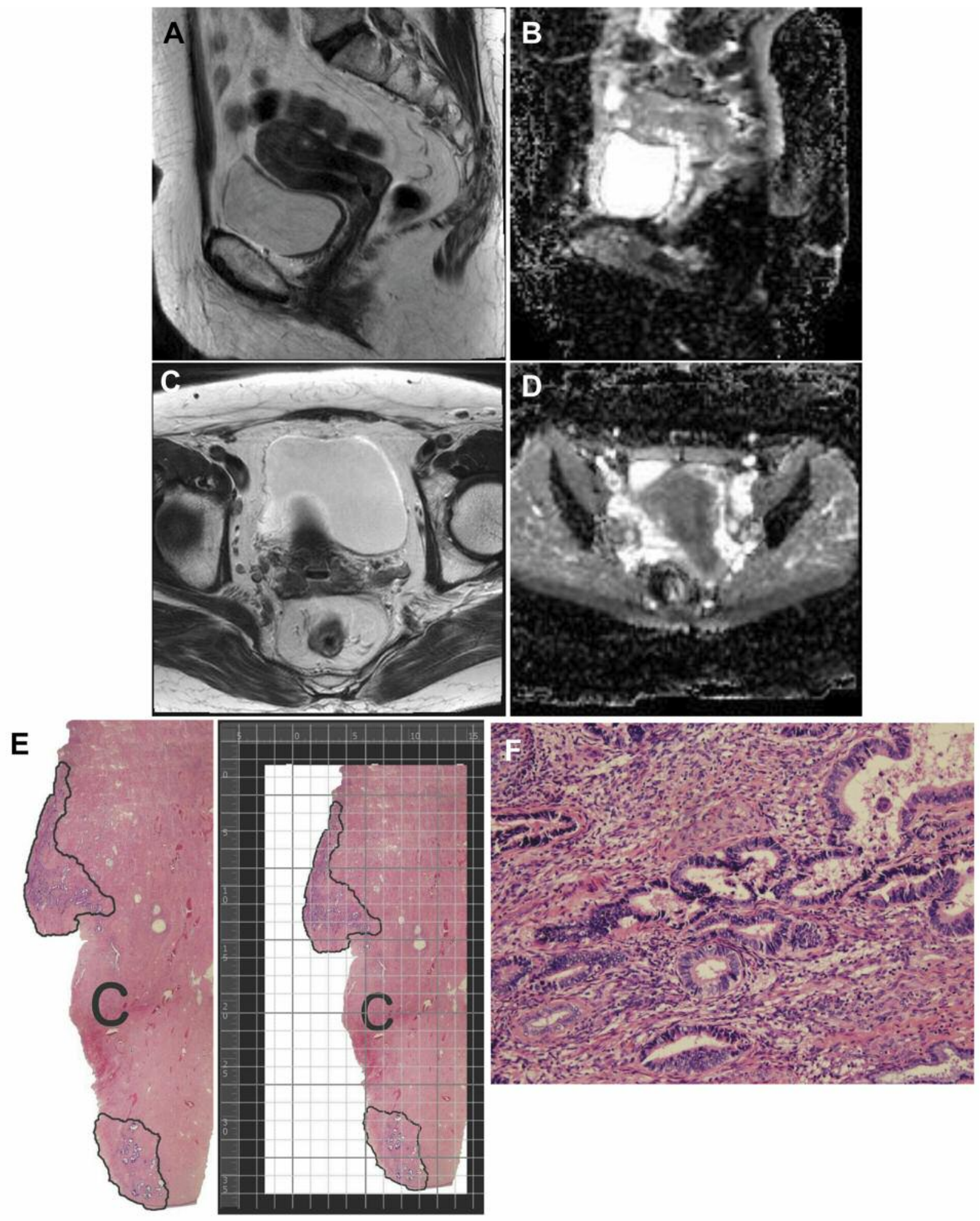

Figure 3. 2009 FIGO Stage IB1 cervical adenocarcinoma in a 40-year-old woman with LEEP prior to MRI (A) sagittal T2-w image and (B) axial T2-w images, $(C)$ b-800 s/mm $\mathrm{mm}^{2} \mathrm{DW}(E)$ sagittal ADC map images, and $(F)$ axial ADC map image show no gross cancerous lesion of the uterine

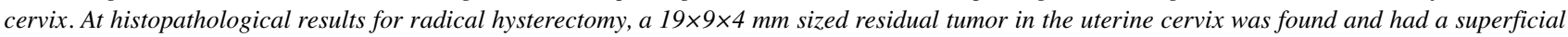
stromal invasion, and (E) Longitudinal section showing a tumor-to-internal os distance was photographed, and $3 \mathrm{~mm}$ calculated. $(F)$ The tumor is well-differentiated, with infiltrative glands. The neoplastic epithelium shows pseudostratified architecture with elongated and enlarged nuclei. Furthermore, it reveals apical mucin depletion and necrotic debris within gland spaces. Mitotic nuclear activity tends to be low. C: Cervical wall. 


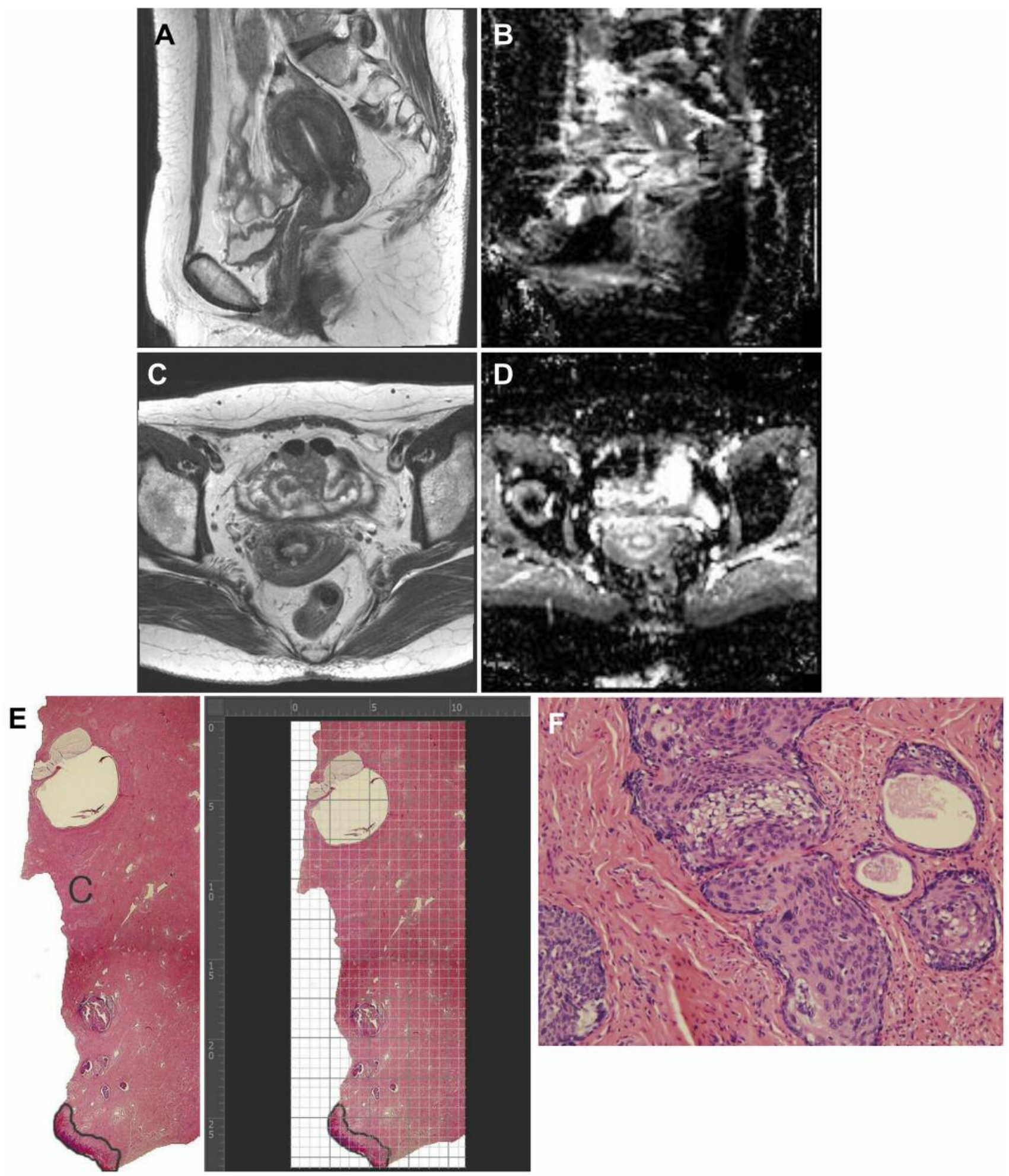

Figure 4. 2009 FIGO Stage IB1 cervical squamous cell carcinoma in a 60-year-old woman who underwent LEEP prior to MRI. (A) sagittal T2-w image and $(B)$ axial T2-w images, $(C)$ b-800 s/mm $\mathrm{mm}^{2} \mathrm{DW}(E)$ sagittal ADC map images, and $(F)$ axial ADC map image show no gross cancerous lesion of the uterine cervix. At histological results for radical hysterectomy, $9 \times 7 \times 4 \mathrm{~mm}$ residual tumor in the uterine cervix was found and had a superficial stromal invasion, and E) Longitudinal section showing a tumor-to-internal os distance was photographed, and $25 \mathrm{~mm}$ calculated. (F) The tumor shows infiltrative sheets and nets of cells. Tumor cells are polygonal squamous cells with occasionally eosinophilic cytoplasm, and mitotic nuclear activity tends to be low. C: Cervical wall. 


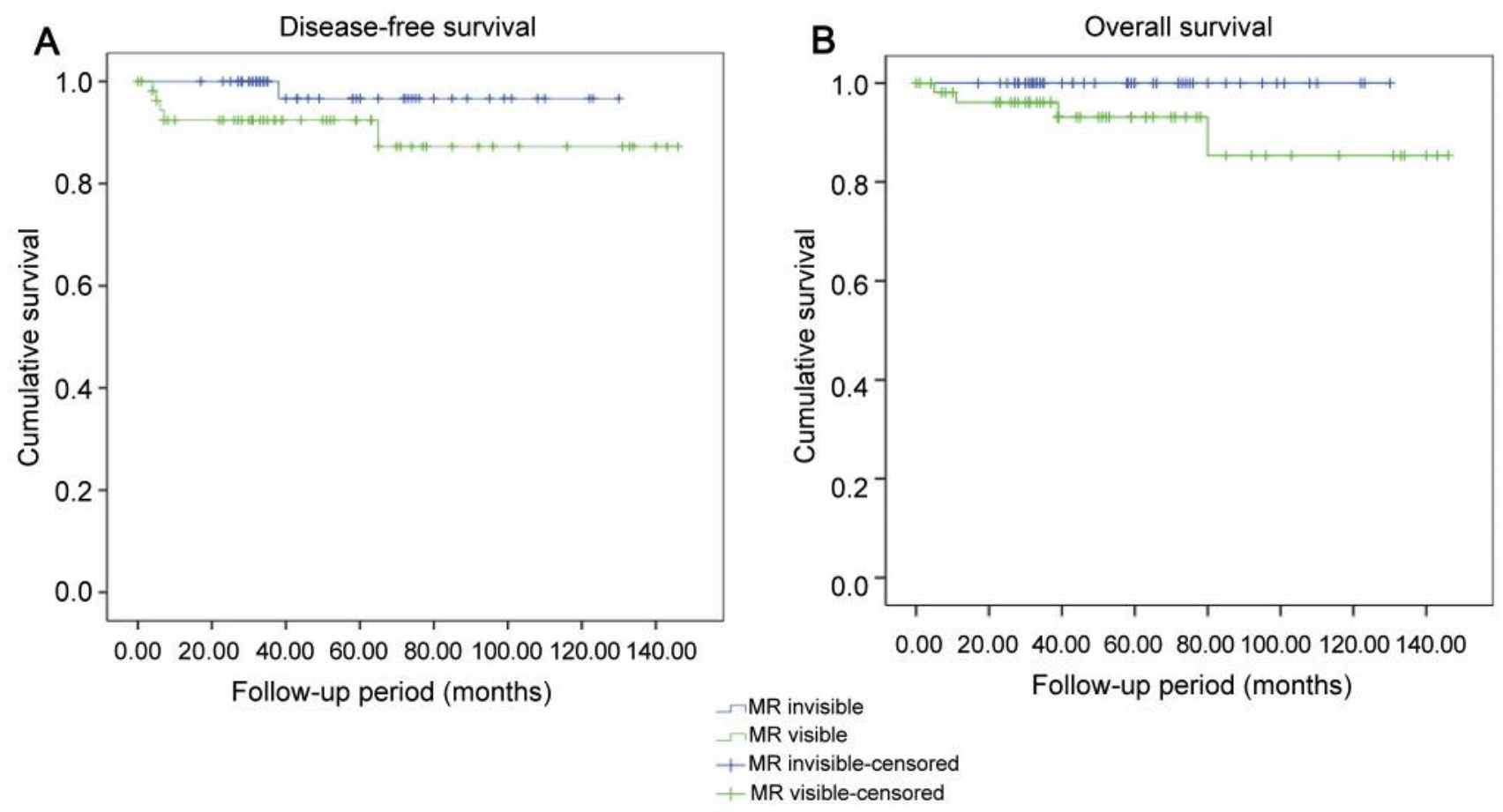

Figure 5. Kaplan-Meier survival curves of magnetic resonance (MR)-invisible and MR-visible cervical cancers. (A) Recurrence-free survival rates of MR-invisible and MR-visible cervical cancers are $98.1 \%$ (53/54 cases) and $91.1 \%$ (51/56 cases), respectively ( $p=0.058)$. (B) OS rates of MRinvisible and MR-visible cervical cancers are 100\% (54/54 cases) and 92.9\% (52/56 cases), respectively $(p=0.048)$.

In a previous publication by Park et al. using the external array coil, MR-invisible stage IB1 disease accounted for $25 \%$ of stage IB1 (FIGO 2009 staging), and $58.1 \%$ of patients had no residual tumor among those with MRinvisible IB1 disease (5).

An early publication by Downey K et al. in 2013 has reported that $\mathrm{T} 2-\mathrm{W}$ plus DWI endovaginal MR-invisible disease accounted for 39\% (22/57) of early-stage IA2-IB1 cervical cancer diagnoses. Of the 22 patients with MRinvisible disease, 21 patients (except for 1 patient due to the lack of histology from the primary site in the patient treated with chemoradiotherapy) with final surgical specimens had results of 19TNs and 2FNs, respectively. Additionally, the authors demonstrated that endovaginal MRI altered surgical management decisions in 12 cases $(38.7 \%)$ planned to receive radical vaginal trachelectomy (10 with extended cone-biopsy and two with chemoradiotherapy) (4).

As opposed to the aforementioned study results, this study showed high false-negative results in 54 MR-invisible patients (17FN with and 14FN without LEEP/conization) regardless of LEEP/conization procedure.

When we consider the inclusion criteria above regarding radical trachelectomy, up to $18.5 \%$ of patients with MRinvisible diagnosis may convert to a more radical treatment than radical trachelectomy.
Park et al. in 2014 have reported that the recurrence-free rates were $98.8 \%$ (85/86) for MR-invisible stage IB1 and 91.2\% (237 of 260) for MR-visible stage IB1 $(p<0.011)$, and the overall 5-year survival rates were $100 \%(86 / 86)$ and $95.8 \%(249 / 260)$, respectively $(p=0.045)(5)$.

In this study, the recurrence-free and overall survival rates in the MR-invisible and MR-visible groups were $98.1 \%$ $(53 / 54)$ vs. $91.1 \%(51 / 56)$ and $100 \%(54 / 54)$ vs. $92.9 \%$ (52/56 cases) $(p=0.058, p=0.048)$, respectively.

The recurrence-free and overall long-term survival rates of the MR-invisible diagnosis were better than those of the MR visible diagnosis.

For patients who did not consider fertility-sparing surgery and had lesions too small to be detected by non-endovaginal imaging, an FN may not affect the clinical survival outcome.

Indeed, the most common cause of misinterpretation between MRI and histological findings after LEEP/conization is due to distortion of the cervix from the following factors: i) blood and air within the potential surgical defects; ii) post-biopsy changes (granulation and scarring post-procedure such as edema, granulation tissue, and fibrosis); and iii) peritumoral inflammatory tissue, which is the most common cause of misinterpretation due to the discrepancy between MRI and histological findings (11). 
The appearance of such factors may create errors in interpretation. Therefore, improving the spatial resolution of MRI to better visualize detailed anatomical structures as well as unwanted features, such as susceptibility artifacts in vivo, is essential and can be implemented with high-ultra field MR using dedicated multichannel coils for clinical access.

Strengths and limitations. There are several limitations to this study. First, selection bias may have been induced, considering the study's retrospective nature. Second, all studies were reviewed by a single radiologist, who had a disadvantage of interobserver variation. Third, the difference in the diagnostic accuracy between T2-WI and T2-WI plus DWI for detecting tumors was not analyzed. Fourth, these results were not validated in an independent setting. Finally, to the best of our knowledge, a comparison of the effectiveness of the transvaginal surface coil and pelvic phased-array coil for evaluating these factors has not been reported.

\section{Conclusion}

The results strongly suggest that due to the high possibility of underdiagnosis for MR-invisible disease, clinicians should consider with caution when choosing between trachelectomy, and radical hysterectomy as well as the safety of each surgical method.

\section{Conflicts of Interest}

The Authors declare that there are no conflicts of interest regarding this study.

\section{Authors' Contributions}

Conceived and designed the experiments: Hyun Jin Roh, Eun Byeol Go, Kyung Bin Kim, Jong Hwa Lee, Sang Hun Lee; Analyzed the data: Hyun Jin Roh, Eun Byeol Go, Kyung Bin Kim, Jong Hwa Lee, Sang Hun Lee; Wrote the paper: Sang Hun Lee; Approval of final version of the manuscript: Hyun Jin Roh, Eun Byeol Go, Kyung Bin Kim, Jong Hwa Lee, Sang Hun Lee.

\section{References}

1 deSouza NM, Soutter WP, McIndoe GA, Gilderdale DJ and Krausz T: Stage I cervical cancer: Tumor volume by magnetic resonance imaging of screen-detected versus symptomatic lesions. J Natl Cancer Inst 89(17): 1314-1315, 1997. PMID: 9293923. DOI: 10.1093/jnci/89.17.1314

2 Bhatla N and Denny L: Figo cancer report 2018. Int J Gynaecol Obstet 143: 2-3, 2018. PMID: 30306587. DOI: 10.1002/ ijgo. 12608

3 Schmeler KM, Frumovitz M and Ramirez PT: Conservative management of early stage cervical cancer: Is there a role for less radical surgery? Gynecol Oncol 120(3): 321-325, 2011. PMID: 21320670. DOI: 10.1016/j.ygyno.2010.12.352
4 Downey K, Jafar M, Attygalle AD, Hazell S, Morgan VA, Giles SL, Schmidt MA, Ind TE, Shepherd JH and deSouza NM: Influencing surgical management in patients with carcinoma of the cervix using a t2- and zoom-diffusion-weighted endovaginal mri technique. Br J Cancer 109(3): 615-622, 2013. PMID: 23868012. DOI: $10.1038 /$ bjc.2013.375

5 Park JY, Lee JW, Park BK, Lee YY, Choi CH, Kim TJ, Bae DS, Kim BG, Park JJ, Park SY and Kim CK: Postoperative outcomes of mr-invisible stage IB1 cervical cancer. Am J Obstet Gynecol 211(2): 168.e161-167, 2014. PMID: 24607752. DOI: 10.1016/j.ajog.2014.02.032

6 deSouza NM, Dina R, McIndoe GA and Soutter WP: Cervical cancer: Value of an endovaginal coil magnetic resonance imaging technique in detecting small volume disease and assessing parametrial extension. Gynecol Oncol 102(1): 80-85, 2006. PMID: 16427688. DOI: 10.1016/j.ygyno.2005.11.038

7 Charles-Edwards E, Morgan V, Attygalle AD, Giles SL, Ind TE, Davis $\mathrm{M}$, Shepherd J, McWhinney $\mathrm{N}$ and deSouza NM: Endovaginal magnetic resonance imaging of stage $1 \mathrm{~A} / 1 \mathrm{~B}$ cervical cancer with a t2- and diffusion-weighted magnetic resonance technique: Effect of lesion size and previous cone biopsy on tumor detectability. Gynecol Oncol 120(3): 368-373, 2011. PMID: 21093895. DOI: 10.1016/j.ygyno.2010.10.013

8 Downey K, Shepherd JH, Attygalle AD, Hazell S, Morgan VA, Giles SL, Ind TE and Desouza NM: Preoperative imaging in patients undergoing trachelectomy for cervical cancer: Validation of a combined t2- and diffusion-weighted endovaginal mri technique at 3.0 t. Gynecol Oncol 133(2): 326-332, 2014. PMID: 24582988. DOI: $10.1016 /$ j.ygyno.2014.02.026

9 Merkle EM and Dale BM: Abdominal MRI at 3.0 t: The basics revisited. AJR Am J Roentgenol 186(6): 1524-1532, 2006. PMID: 16714640. DOI: 10.2214/AJR.05.0932

10 Dietrich O, Reiser MF and Schoenberg SO: Artifacts in 3-t MRI: Physical background and reduction strategies. Eur J Radiol 65(1): 29-35, 2008. PMID: 18162353. DOI: 10.1016/j.ejrad. 2007.11.005

11 deSouza NM, Scoones D, Krausz T, Gilderdale DJ and Soutter WP: High-resolution mr imaging of stage I cervical neoplasia with a dedicated transvaginal coil: MR features and correlation of imaging and pathologic findings. AJR Am J Roentgenol 166(3): 553-559, 1996. PMID: 8623627. DOI: 10.2214/ajr.166.3.8623627

12 Soutter WP, Hanoch J, D'Arcy T, Dina R, McIndoe GA and DeSouza NM: Pretreatment tumour volume measurement on high-resolution magnetic resonance imaging as a predictor of survival in cervical cancer. Bjog 111(7): 741-747, 2004. PMID: 15198766. DOI: 10.1111/j.1471-0528.2004.00172.x

13 Shin SJ, Kim KR, Song DE, Ro JY, Kong KY, Lee SW and Nam JH: Recognition of parametrial invasion, an important landmark when treating cervical cancer. Gynecol Oncol 124(3): 502-507, 2012. PMID: 22108255. DOI: 10.1016/j.ygyno.2011.11.015

14 Meigs JV and Brunschwig A: A proposed classification for cases of cancer of the cervix treated by surgery. Am J Obstet Gynecol 64(2): 413-415, 1952. PMID: 14952540. DOI: 10.1016/00029378(52)90319-0

15 Van Nagell JR, Jr., Roddick JW Jr. and Lowin DM: The staging of cervical cancer: Inevitable discrepancies between clinical staging and pathologic findinges. Am J Obstet Gynecol 110(7): 973-978, 1971. PMID: 5558978. DOI: 10.1016/0002-9378(71)90551-5

16 Lakhman Y, Akin O, Park KJ, Sarasohn DM, Zheng J, Goldman DA, Sohn MJ, Moskowitz CS, Sonoda Y, Hricak H and Abu- 
Rustum NR: Stage IB1 cervical cancer: Role of preoperative MR imaging in selection of patients for fertility-sparing radical trachelectomy. Radiology 269(1): 149-158, 2013. PMID: 23788721. DOI: $10.1148 /$ radiol.13121746

17 Downey K, Attygalle AD, Morgan VA, Giles SL, MacDonald A, Davis M, Ind TE, Shepherd JH and deSouza NM: Comparison of optimised endovaginal $v s$. external array coil t2-weighted and diffusion-weighted imaging techniques for detecting suspected early stage (IA/IB1) uterine cervical cancer. Eur Radiol 26(4): 941-950, 2016. PMID: 26162579. DOI: 10.1007/s00330-0153899-5

18 Fischerova D, Cibula D, Stenhova H, Vondrichova H, Calda P, Zikan M, Freitag P, Slama J, Dundr P and Belacek J: Transrectal ultrasound and magnetic resonance imaging in staging of early cervical cancer. Int J Gynecol Cancer 18(4): 766-772, 2008. PMID: 17892456. DOI: 10.1111/j.1525-1438.2007.01072.x

19 Sonoda Y, Chi DS, Carter J, Barakat RR and Abu-Rustum NR: Initial experience with dargent's operation: The radical vaginal trachelectomy. Gynecol Oncol 108(1): 214-219, 2008. PMID: 17996284. DOI: 10.1016/j.ygyno.2007.09.028
20 Leblanc E, Narducci F, Ferron G and Querleu D: Indications and teaching of fertility preservation in the surgical management of gynecologic malignancies: European perspective. Gynecol Oncol 114: S32-36, 2009. PMID: 19573704. DOI: 10.1016/ j.ygyno.2009.04.010

21 Noel P, Dube M, Plante M and St-Laurent G: Early cervical carcinoma and fertility-sparing treatment options: MR imaging as a tool in patient selection and a follow-up modality. Radiographics 34(4): 1099-1119, 2014. PMID: 25019444. DOI: 10.1148/rg.344130009
Received October 29, 2019

Revised November 11, 2019

Accepted November 13, 2019 